\title{
Identification and characterization of the Nep1-like protein UvNLP in rice false smut fungus Ustilaginoidea virens
}

\section{Yufu Wang}

Huazhong Agricultural University College of Plant Science and Technology Jinsong Qu

Huazhong Agricultural University College of Plant Science and Technology

Yueran Liu

Huazhong Agricultural University College of Plant Science and Technology

\section{Geng Sun}

Huazhong Agricultural University College of Plant Science and Technology

\section{Zuoqian Wang}

Huazhong Agricultural University College of Plant Science and Technology

\section{Liangfen Yin}

Huazhong Agricultural University College of Plant Science and Technology

\section{Weixiao Yin ( $\nabla$ wxyin@mail.hzau.edu.cn )}

Huazhong Agricultural University College of Plant Science and Technology https://orcid.org/00000002-5231-3979

\section{Chaoxi Luo}

Huazhong Agricultural University College of Plant Science and Technology

\section{Research article}

Keywords: Ustilaginoidea virens, rice false smut, UvNLP, cell death, plant defence

Posted Date: January 3rd, 2020

DOl: https://doi.org/10.21203/rs.2.19940/v1

License: (c) (i) This work is licensed under a Creative Commons Attribution 4.0 International License. Read Full License 


\section{Abstract}

Background The necrosis- and ethylene-inducing peptide 1 (Nep1)-like proteins (NLPs) are a broadly distributed superfamily of protiens in plant-associated microorganisms, including bacteria, fungi, and oomycetes. NLPs are considered to be important virulence factors, and some have been well studied. However, the role of NLPs in Ustilaginoidea virens, the agent of rice false smut fungus, remains to be uncovered.

Results A protein containing NLP-specific NPP1 domain was identified in U. virens and named UvNLP. Phylogenetic analysis revealed that UvNLP was a type 2 NLP, and real-time PCR revealed that UvNLP was highly expressed at 3 days post-inoculation. A yeast secretion assay demonstrated that UvNLP contained a functional signal peptide (SP). Furthermore, transient expression of UvNLP in Nicotiana benthamiana induced weak cell death, and replacement of the SP enhanced cell death. Real-time PCR indicated that UvNLP induced the expression of plant defence related genes.

Conclusions We identified a secreted protein UvNLP in U. virens that was infection related and could induce plant cell death and defence responses. This study provided evidence that NLPs act as proteinaceous MAMPs, giving insight into the elucidation of the pathogenicity mechanism of $U$. virens. The UvNLP might be used as a potential plant defence inducer for disease control in practice.

\section{Background}

Many microbial pathogens secrete an abundance of proteins to subvert host immunity, remodel cellular functions and promote pathogen proliferation [1-4]. The effector functions can be analyzed in the context of the "zigzag" model of a two-layered immune system in plants [5]. The first layer is pattern recognition receptors (PRRs), which detect microbial/pathogen-associated molecular patterns (MAMPs/PAMPs) to trigger MAMP/PAMP-triggered immunity (MTI or PTI) $[5,6]$. The second layer is the effectors recognized by NB-LRR proteins, activating effector-triggered immunity (ETI) [5].

Among bacterial MAMPs, flagellin, EF-Tu and peptidoglycan have been well characterized [7-9]. Chitin and xylanase are important fungal MAMPs $[10,11]$. The secreted polygalacturonase in Botrytis cinerea was identified as a MAMP that triggered plant immunity [12]. Several MAMPs from oomycetes have been identified and can trigger immunity, e.g., INF1, CBEL, Crinklers (CRN) and cytotoxic necrosis and NLPs [13]. Recently, XEG1 from Phytophthora sojae was identified as a PAMP that was specifically recognized by the LRR receptor-like protein RXEG1 $[14,15]$.

The 24-kDa Nep1 was firstly identified from the plant pathogenic fungus Fusarium oxysporum in culture filtrates and shown to induce necrosis and ethylene production when it was infiltrated into the extracellular space of the leaves of dicot but not monocot plants [16, 17]. The Nep1-like proteins constitute a superfamily produced by plant pathogenic bacteria, fungi and oomycetes, and they include three major types. The vast majority of NLPs have a SP and an NPP1 domain containing a heptapeptide motif, GHRHDWE [18]. Many NLPs are necrotizing cytolytic toxins, and they have structural similarities to 
actinoporins, which are pore-forming toxins from sea anemones [19]. NLPs generally cause necrosis or cell death only when they contain a SP, suggesting that the NLP acts in the extracellular space of the plant to confer its cytotoxic activity [20,21]. In addition to causing necrosis, NLPs also stimulate defence responses, indicating important roles during plant-pathogen interactions $[17,19]$. An immunogenic pattern of 24 amino acid residues of NLPs triggered plant defence in a manner similar to the prototype pattern, bacterial flagellin [22, 23]. In addition to cytotoxic NLPs, many noncytotoxic NLPs that do not induce necrosis have been described [24-27]. NLPs may disrupt the membrane to induce necrosis, thus leading to plant immune and cell death responses $[23,28]$. Glycosyl inositol phosphorylceramide (GIPC) sphingolipids were identified as NLP toxin receptors. The reason that monocot plants are insensitive to NLP cytolysins may be explained by the length of the GIPC head group. Monocot GIPCs have three hexoses that prevent the NLP from inserting into the plant membrane, whereas eudicot GIPCs have only two hexoses, allowing the NLP to contact and insert into the lipid bilayer $[29,30]$.

Although NLPs are widespread across microorganisms, the number of NLP family members varies significantly. Thirty-three and thirty-nine confirmed NLPs were identified in P. sojae and P. capsici, respectively, some of which can induce necrosis, while some cannot [25,31]. A few NLPs have been found in fungal genomes. Only a single NLP (MgNLP) was found in the wheat pathogen Mycosphaerella graminicola. Heterologously expressed MgNLP protein induces necrotic cell death and the activation of defence-related genes in Arabidopsis but not in susceptible wheat. Targeted deletion of MgNLP did not cause detectable reduction in plant pathogenicity or virulence [32]. Two of the nine NLPs from Verticillium dahlia (V592) induced necrotic lesions and triggered defence responses in N. benthamiana, Arabidopsis, and cotton plants, and both contributed to pathogenicity in tomato and Arabidopsis but were dispensable in cotton [24, 33]. Four MoNLPs were identified in Magnaporthe oryzae, three of which could trigger the accumulation of reactive oxygen species and cell death but were dispensable for pathogenicity on rice plants [34].

In this study, we identified a single NLP-specific NPP1 domain containing protein, UvNLP, in U. virens, the causal agent of rice false smut. UvNLP was a type 2 NLP and showed high expression at early infection. UvNLP contains a functional SP and induces cell death and defence response in plants. Our results provide a potential elucidation of the $U$. virens pathogenicity mechanism and evidence for the functional diversification of NLPS.

\section{Results}

\section{Identification of the single NPP1 domain-containing protein (UvNLP) in $U$. virens}

The genome data of $U$. virens have been released, which may enhance the systematic identification and analysis of many important proteins [35]. To investigate the composition of the NLP family in U. virens, a HMMER alignment search was performed on the proteome of $U$. virens with the hidden Markov model (HMM) profile of the NLP-specific NPP1 domain (PF05630), and three candidate proteins were found (KDB14180, KDB15463 and KDB11767). Combining the $E$ value and conformation using SMART 
programme, only a single NPP1 domain-containing protein, KDB14180, was identified in $U$. virens; we named it UvNLP. The UvNLP protein contains 302 amino acids, including eight cysteine residues and a predicted signal peptide (Fig. 1 A). Previous research has shown that NLPs can be divided into three types [28]. According to the phylogenetic analysis, UvNLP belongs to type 2 (Fig. 1 B) and showed high similarity with NLP from Metarhizium acridum, consistent with the corresponding evolutionary relationship.

UVNLP is highly expressed at the early stage of infection

To determine the potential role of UvNLP during the infection of $U$. virens, a high-virulence isolate was inoculated into the panicles of a susceptible rice cultivar. The samples at $3,5,6$ and 7 days postinoculation (dpi) were collected to analyze the transcript level by qRT-PCR. Result showed that UvNLP was strongly induced at $3 \mathrm{dpi}$ and then the expression level decreased gradually over time. Because the hyphae entered the spikelets and reached the surface of the floral organs of rice at $3 \mathrm{dpi}$ [36], these results indicated that UVNLP was involved in infection at an early stage (Fig. 2).

UvNLP contains a functional signal peptide

The amino acid sequence analysis showed that UvNLP contained a predicted signal peptide. To validate the function of the predicted signal peptide, the yeast system was selected for experimental verification. The nucleotide sequence coding the predicted signal peptide and the following three amino acids was fused into the vector pSUC2, which was subsequently transformed into the YTK12 strain. The SP of Avr1b from $P$. sojae and the pSUC2 empty vector were used as positive and negative controls. Results showed that the yeast containing the pSUC2 empty vector, Avr1b and UvNLP, were able to grow on CMD$W$ medium, suggesting that the vectors were successfully transformed into yeast. In addition, the yeast containing Avr1b and UvNLP constructs grew normally on YPRAA medium, indicating that the SPs in both constructs were functional. Moreover, invertase secretion was further confirmed with an enzymatic activity test based on reduction of the dye 2,3,5-triphenyltetrazolium chloride (TTC) to the insoluble redcoloured triphenylformazan. In contrast, the empty vector control yeast strains did not grow normally on YPRAA, and the TTC-treated culture filtrates remained colourless (Fig. 3). Despite the lighter red was observed, all these results indicate that the signal peptide of UvNLP is functional, and thus, it is a secreted protein.

UvNLP induces weak cell necrosis in N. benthamiana

Previous studies have mentioned that certain NLPs could induce necrosis upon infiltration into the leaves of dicotyledonous plants. To determine the necrosis-inducing activity of UvNLP, the full-length gene was 
constructed into the vector for $A$. tumefaciens-mediated plant expression. Significant cell death was not observed after 7 days of UvNLP expression in $N$. benthamiana. Further observation revealed weak cell death (Fig. 4). The protein expression was detected by western blot, and the results showed that all of the proteins were expressed. This result indicated that UVNLP has cytotoxic activity in N. benthamiana.

A weaker red colour was observed for UvNLP SP than for Avr1b SP in the TTC assay, which may indicate a weaker secretory ability. To investigate whether the function of SP is related to cell death, the UvNLP SP was replaced by Avr1b SP and then transiently expressed in $N$. benthamiana. Significant cell death was observed (Fig. 4), indicating that the weak cell death was related to UvNLP signal peptide secretion but not the UvNLP itself.

UvNLP induces genes expression involved in defence responses in $N$. benthamiana

In addition to inducing cell death, NLP proteins could trigger plant immunity or a defence response [22, 23]. To investigate whether UvNLP induces the expression of defence-related genes, the expression of related genes was detected after UvNLP infiltration. The results showed that UvNLP induced the expression of ethylene-responsive element binding factors 5 (ERF5) and 1-aminocyclopropane-1carboxylate (ACC) synthase (ACS1 and ACS3) related to ethylene biosynthesis. Respiratory burst oxidase homologue protein $\mathrm{B}(R b o h B)$, a calcium-dependent NADPH oxidase that generates superoxide, was also induced significantly. Moreover, UvNLP treatment triggered the plant defence-related genes WRKYG and P450 (Fig. 5). Therefore, all the results indicated that, in addition to inducing cell death, UvNLP elicits a plant defence response.

\section{Discussion}

In this study, a single NPP1 domain-containing NLP family member, UvNLP, was identified in U. virens and shown to contain a functional signal peptide. Notably, its conserved heptapeptide motif was "GHQYDWE" instead of "GHRHDWE", which is consistent with the research that the two dissimilar amino acids were less conserved [28]. The phylogenetic clustering shows that UvNLP belongs to the type 2 NLPs, which are widely distributed in fungi and bacteria with various lifestyles, including plant- or non-plant-associated microbes and insect-pathogenic fungi [28].

The expression of NLP genes has been described in plant-pathogen interactions. In the obligate biotroph oomycete Hyaloperonospora arabidopsidis, four HaNLPs were induced at the early stage of infection. Four NLPs from P. capsici were active during the early infection stage, and three were expressed only at later stages [31]. MgNLP, the only NLP gene in the hemibiotroph M. graminicola, is highly expressed specifically towards the end of the symptomless stage of the infection process [32]. In the necrotrophic fungal pathogen B. cinerea, BcNep1 and BcNep2 were induced at the early and late stages of infection, respectively, suggesting that they play different roles during pathogenesis [37]. All these studies indicated that NLPs were involved in the pathogen-plant interaction, and their expression patterns implied that 
NLPs may have diverse roles during infection. We observed that the transcript level of UvNLP was highest at 3 dpi during the infection process, indicating a function at the early infection stage, because $3 \mathrm{dpi}$ is the initial contact time for $\mathrm{U}$. virens hyphae extending to rice floral organs [36].

Although NLPs were initially identified to induce necrosis in plants [18, 21], NLPs that did not induce necrosis were subsequently found in abundance. Among the VdNLPs in V. dahliae, two of the nine NLPs had the ability to induce cell death and trigger immunity-associated responses in $\mathrm{N}$. benthamiana, Arabidopsis, and cotton plants [24]. Two of the three tested NLPs in P. infestans and eleven of the nineteen NLPs in P. sojae did not cause necrosis when transiently expressed in plants [25,38]. None of the twelve NLPs (HaNLPs) identified in H. arabidopsidis cause necrosis [26]. However, HaNLP3 activated the expression of a large set of defence-related genes and enhanced resistance to $\mathrm{H}$. arabidopsidis when ectopically expressed in Arabidopsis [23]. We found that UvNLP induced weak cell death when expressed in $\mathrm{N}$. benthamiana, indicating cytotoxic activity.

The NLPs have an N-terminal SP, indicating that they are secreted into the extracellular space of plants $[21,28]$. This is shown by the PsojNIP, which caused necrosis when it included its SP [20]. In this study, we found that UVNLP contained a predicted SP, which was demonstrated to be functional, although secretion function was weak. The UvNLP that SP was replaced by which from Avr1b induced significant cell death, indicating secretion function played an important role for UvNLP to induce cell death. In H. arabidopsidis, an exposed domain prevents HaNLP3 from inducing cell death [26].

We further investigated the expression of defence-related genes when expressed UvNLP and found that some genes were induced. We proposed that UvNLP might not only act as an elicitor but also play other roles in contributing to the infection of $U$. virens. Thus, further investigation of UvNLP with noncytolytic assays should be conducted. On the other hand, because of its ability of triggering plant defence, UvNLP has the potential to be a plant defence inducer for pathogen control in practice.

\section{Conclusions}

We identified the single NLP protein UvNLP in U. virens, which contains a functional signal peptide and shows a high expression level at $3 \mathrm{dpi}$, indicating a potentially significant role for the infection. Transient expression of UvNLP in plants induces weak cell death and the expression of plant defence-related genes. Our study provides evidence that NLPs may act as proteinaceous MAMPs, giving insight into the elucidation of the U. virens pathogenicity mechanism. The UvNLP might be used as a potential plant defence inducer for disease control in practice.

\section{Methods}

\section{NLP identification and bioinformatics analysis}

Hidden Markov model (HMM) profile searches were performed against the $U$. virens protein database downloaded from the National Center for Biotechnology site (GenBank accession number 
JHTR00000000) [35] using an NPP1 domain (Pfam: PF05630) as a query. The signal peptide and NPP1 were predicted with SMART programme (http://smart.embl-heidelberg.de/ Accessed 05 Dec 2019)[39]. The phylogenetic tree was constructed using the MEGA 5 program.

\section{Growth conditions of plants and bacteria, etc.}

benthamiana was grown in a plant growth room at $25^{\circ} \mathrm{C}$ with $14 \mathrm{~h}$ lights and $10 \mathrm{~h}$ dark. The yeast YTK12 strain was cultured at $30^{\circ} \mathrm{C}$. Agrobacterium tumefaciens strain GV3101 was cultured in LB (LuriaBertani) medium at $30{ }^{\circ} \mathrm{C}$ for transient expression.

\section{Nucleic acid isolation and real-time PCR analysis}

To monitor UvNLP transcript profiling during $U$. virens infection, the rice panicles were inoculated as described previously [36]. For the detection of resistance-related genes in $N$. benthamiana, the leaves were harvested at 3 and 5 days post-infiltration (dpi). Total RNA samples were isolated using TRIzol, and the cDNA was synthesized using a Thermo Scientific RevertAid First Strand cDNA Synthesis Kit (Thermo Fisher Scientific, Waltham, MA). The reaction mixtures were diluted 6 times with distilled water and used as templates for quantitative real-time polymerase chain reaction (qRT-PCR). Gene amplification was performed in a CFX96 Real-Time PCR Detection System (Bio-Rad Laboratories Inc., Hercules, CA) using SYBR Green I fluorescent dye. The $\beta$-tubulin gene served as an internal control for the expression studies.

\section{Molecular cloning and plasmid construction}

The full-length UVNLP gene, including the signal peptide-encoding region, was amplified from cDNA of the $U$. virens infected rice. The PCR products were digested with Cla I and Sma I and cloned into the vector pGR107. To validate the function of the SP, the signal peptide-encoding region was amplified and cloned directly into the vector pSUC2. The SP of Avr1b and UvNLP without SP were amplified, respectively and fused by using overlap PCR. The fragments were inserted into the vector by using the ClonExpress MultiS One Step Cloning Kit (Vazyme, Nanjing, China). The sequences were showen in Additional file 1. The recombinant clones with correct orientation were identified and confirmed by sequencing.

\section{Validation of predicted signal peptide}

The pSUC2-derived plasmids were transformed into the invertase-negative yeast strain YTK12 using the lithium acetate method [40]. The yeast strains were then plated on CMD-W plates $(0.67 \%$ yeast $\mathrm{N}$ base without amino acids, $0.075 \%$ tryptophan dropout supplement, $2 \%$ sucrose, $0.1 \%$ glucose, and $2 \%$ agar). The transformed colonies were transferred to fresh CMD-W and YPRAA (1\% yeast extract, $2 \%$ peptone, $2 \%$ raffinose and $2 \mathrm{mg} / \mathrm{mL}$ antimycin A) plates and incubated at $30{ }^{\circ} \mathrm{C}$. The enzymatic activity of invertase was detected by the reduction of TTC to insoluble red-coloured triphenylformazan as described previously [41]. The yeast transformants were inoculated in 2 to $3 \mathrm{~mL}$ of YPDA (1\% yeast extract, $2 \%$ peptone, $2 \%$ glucose, $0.03 \%$ adenine hemisulfate and $2 \%$ agar) medium and incubated for $24-36 \mathrm{~h}$ at 30 ${ }^{\circ} \mathrm{C}$. Then, $1.5 \mathrm{~mL}$ yeast was collected by centrifugation and resuspended in $750 \mu \mathrm{L}$ sterilized water. Then, $250 \mu \mathrm{L}$ of acetic acid-sodium acetate buffer $(10 \mathrm{mM}, \mathrm{pH}=4.7)$ and $500 \mu \mathrm{L}$ of sucrose solution $(10 \% \mathrm{w} / \mathrm{v})$ 
were added and incubated at $37^{\circ} \mathrm{C}$ for $10 \mathrm{~min}$. The supernatant $(100 \mu \mathrm{L})$ was collected after centrifugation, mixed with $900 \mu \mathrm{L}$ of $0.1 \%$ TTC and incubated at room temperature for $5 \mathrm{~min}$. A colorimetric change was observed if the signal peptide was functional.

\section{Agroinfiltration and western blot assay}

For agroinfiltration, the $A$. tumefaciens strains were cultured in LB medium containing $50 \mu \mathrm{g} / \mathrm{mL}$ kanamycin with shaking for $24 \mathrm{~h}$. The cultures were harvested by centrifuging at $3000 \mathrm{rpm}$ for $5 \mathrm{~min}$, washed with $10 \mathrm{mM} \mathrm{MgCl}_{2}$ three times and resuspended to a final $\mathrm{OD}_{600}$ of 0.4 . Four- to six-week-old $N$. benthamiana leaves were selected for infiltration. A small nick was formed in the leaves with a needle, and an appropriate amount of the cell suspension was then infiltrated using a syringe without the needle. Cell death was observed 7 days after infiltration.

The $N$. benthamiana leaves were harvested and ground in $1 \mathrm{~mL}$ RIPA buffer (Thermo Scientific Pierce; No. 89900) containing $10 \mu \mathrm{L} 100 \mathrm{mM}$ phenylmethylsulfonyl fluoride (Beyotime; ST506) after 2 days of infiltration. The protein extract was fractionated using SDS-PAGE and then transferred onto PVDF membrane (BIO-RAD; 162-0177). The membrane was blocked with $5 \% \mathrm{w} / \mathrm{v}$ non-fat dry milk in TBS (50 $\mathrm{mM}$ Tris- $\mathrm{HCl}, \mathrm{pH} 7.5,150 \mathrm{mM} \mathrm{NaCl}$ ) for $1 \mathrm{~h}$ at room temperature with agitation. The HA-Tag mouse monoclonal antibody (CWBIO; CW0092) was added into TBS containing $5 \%$ milk and incubated at $4{ }^{\circ} \mathrm{C}$ for $12 \mathrm{~h}$, followed by four washes ( 5 min each) in TBST (TBS containing $0.05 \%$ Tween 20). After that, the membrane was incubated with goat anti-mouse IgG-HRP Conjugate (CWBIO; CW0102) at the recommended concentration in TBS containing 5\% milk at room temperature for 30 min with gentle agitation. Mouse anti-FLAG monoclonal antibodies (HRP) (Sigma Aldrich, A8592) were used to detect the protein-fused FLAG tag by adding them into TBS containing $5 \%$ milk and incubating at $4{ }^{\circ} \mathrm{C}$ temperature for $12 \mathrm{~h}$, followed by four washes ( 5 min each) in TBST. The membrane was treated with the Western ECL Substrate (BIO-RAD; 170-5060) for 1 min without agitation. The wrapped blot was then exposed to plastic film for a range of times from several seconds up to 10 min depending on the signal observed.

\section{Abbreviations}

NLP: necrosis- and ethylene-inducing peptide 1 (Nep1)-like protein; PRR: pattern recognition receptor; MAMP/PAMP: microbial/pathogen-associated molecular patterns; MTI or PTI: MAMP/PAMP-triggered immunity; SP: signal peptide; GIPC: glycosyl inositol phosphorylceramide; TTC: 2,3,5-triphenyltetrazolium chloride; ERF5: Ethylene-responsive element binding factors 5; ACC: 1-aminocyclopropane-1-carboxylate; $R b o h B$ : respiratory burst oxidase homologue protein $\mathrm{B}$; dpi: days post-inoculation.

\section{Declarations}

\section{Funding}

This work was supported by the National Natural Science Foundation of China (No. 31701736), National Key Research and Development Programme (2016YFD0300700) and the Fundamental Research Funds 
for the Central Universities (2662017JC003).

\section{Availability of data and materials}

All data and material are available from the authors upon request.

\section{Authors' contributions}

WY and CL conceived and designed the study. YW, WY, JQ, YL, GS, ZW and LY conducted the experiments and analyzed the data. YW, WY and CL prepared the manuscript, and all authors approved the final version of the manuscript.

\section{Ethics approval and consent to participate}

Not applicable.

\section{Consent for publication}

Not applicable.

\section{Competing interests}

The authors declare that they have no competing interests.

\section{References}

1. Lo Presti L, Lanver D, Schweizer G, Tanaka S, Liang L, Tollot M, Zuccaro A, Reissmann S, Kahmann R: Fungal effectors and plant susceptibility. Annu Rev Plant Biol 2015, 66:513-545.

2. Lindeberg M, Cunnac S, Collmer A: Pseudomonas syringae type III effector repertoires: last words in endless arguments. Trends Microbiol 2012, 20(4):199-208.

3. Bozkurt TO, Schornack S, Banfield MJ, Kamoun S: Oomycetes, effectors, and all that jazz. Curr Opin Plant Biol 2012, 15(4):483-492.

4. Kamoun S: A catalogue of the effector secretome of plant pathogenic oomycetes. Annu Rev Phytopathol 2006, 44:41-60.

5. Jones JD, Dangl JL: The plant immune system. Nature 2006, 444(7117):323-329.

6. Zipfel C: Plant pattern-recognition receptors. Trends in Immunology 2014, 35(7):345-351.

7. Felix G, Duran JD, Volko S, Boller T: Plants have a sensitive perception system for the most conserved domain of bacterial flagellin. Plant J 1999, 18(3):265-276.

8. Kunze G, Zipfel C, Robatzek S, Niehaus K, Boller T, Felix G: The N terminus of bacterial elongation factor Tu elicits innate immunity in Arabidopsis plants. Plant Cell 2004, 16(12):3496-3507.

9. Gust AA, Biswas R, Lenz HD, Rauhut T, Ranf S, Kemmerling B, Gotz F, Glawischnig E, Lee J, Felix G et al: Bacteria-derived peptidoglycans constitute pathogen-associated molecular patterns triggering innate immunity in Arabidopsis. Journal of Biological Chemistry 2007, 282(44):32338-32348. 
10. Ron M, Avni A: The receptor for the fungal elicitor ethylene-inducing xylanase is a member of a resistance-like gene family in tomato. Plant Cell 2004, 16(6):1604-1615.

11. Felix G, Regenass M, Boller T: Specific perception of subnanomolar concentrations of chitin fragments by tomato cells - induction of extracellular alkalinization, changes in protein-phosphorylation, and establishment of a refractory state. Plant J 1993, 4(2):307-316.

12. Zhang LS, Kars I, Essenstam B, Liebrand TWH, Wagemakers L, Elberse J, Tagkalaki P, Tjoitang D, van den Ackerveken G, van Kan JAL: Fungal endopolygalacturonases are recognized as microbe-associated molecular patterns by the arabidopsis receptor-like protein RESPONSIVENESS TO BOTRYTIS POLYGALACTURONASES1. Plant Physiol 2014, 164(1):352-364.

13. Hein I, Gilroy EM, Armstrong MR, Birch PR: The zig-zag-zig in oomycete-plant interactions. Mol Plant Pathol 2009, 10(4):547-562.

14. Ma Z, Song T, Zhu L, Ye W, Wang Y, Shao Y, Dong S, Zhang Z, Dou D, Zheng X et al: A Phytophthora sojae glycoside hydrolase 12 protein is a major virulence factor during soybean infection and is recognized as a PAMP. Plant Cell 2015, 27(7):2057-2072.

15. Wang Y, Xu YP, Sun YJ, Wang HB, Qi JM, Wan BW, Ye WW, Lin YC, Shao YY, Dong SM et al: Leucinerich repeat receptor-like gene screen reveals that Nicotiana RXEG1 regulates glycoside hydrolase 12 MAMP detection. Nat Commun 2018, 9:594.

16. Bailey BA: Purification of a protein from culture filtrates of Fusarium oxysporum that induces ethylene and necrosis in leaves of Erythroxylum coca. Phytopathology 1995, 85(10):1250-1255.

17. Gijzen M, Nurnberger T: Nep1-like proteins from plant pathogens: recruitment and diversification of the NPP1 domain across taxa. Phytochemistry 2006, 67(16):1800-1807.

18. Fellbrich G, Romanski A, Varet A, Blume B, Brunner F, Engelhardt S, Felix G, Kemmerling B, Krzymowska M, Nurnberger T: NPP1, a Phytophthora-associated trigger of plant defense in parsley and Arabidopsis. Plant J 2002, 32(3):375-390.

19. Ottmann C, Luberacki B, Kufner I, Koch W, Brunner F, Weyand M, Mattinen L, Pirhonen M, Anderluh G, Seitz HU et al: A common toxin fold mediates microbial attack and plant defense. Proc Natl Acad Sci U S A 2009, 106(25):10359-10364.

20. Qutob D, Kemmerling B, Brunner F, Kufner I, Engelhardt S, Gust AA, Luberacki B, Seitz HU, Stahl D, Rauhut $\mathrm{T}$ et al: Phytotoxicity and innate immune responses induced by Nep1-like proteins. Plant Cell 2006, 18(12):3721-3744.

21. Qutob D, Kamoun S, Gijzen M: Expression of a Phytophthora sojae necrosis-inducing protein occurs during transition from biotrophy to necrotrophy. Plant J 2002, 32(3):361-373.

22. Bohm H, Albert I, Oome S, Raaymakers TM, Van den Ackerveken G, Nurnberger T: A conserved peptide pattern from a widespread microbial virulence factor triggers pattern-induced immunity in Arabidopsis. PLoS Pathog 2014, 10(11):e1004491.

23. Oome S, Raaymakers TM, Cabral A, Samwel S, Bohm H, Albert I, Nurnberger T, Van den Ackerveken G: Nep1-like proteins from three kingdoms of life act as a microbe-associated molecular pattern in Arabidopsis. Proc Natl Acad Sci U S A 2014.

24. Zhou BJ, Jia PS, Gao F, Guo HS: Molecular characterization and functional analysis of a necrosisand ethylene-Inducing, protein-encoding gene family from Verticillium dahliae. Mol Plant Microbe Interact 
2012, 25(7):964-975.

25. Dong S, Kong G, Qutob D, Yu X, Tang J, Kang J, Dai T, Wang H, Gijzen M, Wang Y: The NLP toxin family in Phytophthora sojae includes rapidly evolving groups that lack necrosis-inducing activity. Mol Plant Microbe Interact 2012, 25(7):896-909.

26. Cabral A, Oome S, Sander N, Kufner I, Nurnberger T, Van den Ackerveken G: Nontoxic Nep1-like proteins of the downy mildew pathogen Hyaloperonospora arabidopsidis: repression of necrosis-inducing activity by a surface-exposed region. Mol Plant Microbe Interact 2012, 25(5):697-708.

27. Kleemann J, Rincon-Rivera LJ, Takahara H, Neumann U, van Themaat EVL, van der Does HC, Hacquard S, Stuber K, Will I, Schmalenbach W et al: Sequential delivery of host-induced virulence effectors by appressoria and intracellular hyphae of the phytopathogen Colletotrichum higginsianum. PLoS Pathog 2012, 8(4).

28. Oome S, Van den Ackerveken G: Comparative and functional analysis of the widely occurring family of Nep1-like proteins. Mol Plant Microbe Interact 2014, 27(10):1081-1094.

29. Van den Ackerveken G: How plants differ in toxin-sensitivity. Science 2017, 358(6369):1383-1384. 30. Lenarcic T, Albert I, Bohm H, Hodnik V, Pirc K, Zavec AB, Podobnik M, Pahovnik D, Zagar E, Pruitt R et al: Eudicot plant-specific sphingolipids determine host selectivity of microbial NLP cytolysins. Science 2017, 358(6369):1431-1434.

31. Chen XR, Huang SX, Zhang Y, Sheng GL, Li YP, Zhu F: Identification and functional analysis of the NLP-encoding genes from the phytopathogenic oomycete Phytophthora capsici. Molecular Genetics and Genomics 2018, 293(4):931-943.

32. Motteram J, Kufner I, Deller S, Brunner F, Hammond-Kosack KE, Nurnberger T, Rudd JJ: Molecular characterization and functional analysis of MgNLP, the sole NPP1 domain-containing protein, from the fungal wheat leaf pathogen Mycosphaerella graminicola. Mol Plant Microbe Interact 2009, 22(7):790799.

33. Santhanam P, van Esse HP, Albert I, Faino L, Nurnberger T, Thomma BPHJ: Evidence for functional diversification within a fungal NEP1-like protein family. Mol Plant Microbe Interact 2013, 26(3):278-286. 34. Fang YL, Peng YL, Fan J: The Nep1-like protein family of Magnaporthe oryzae is dispensable for the infection of rice plants. Scientific Reports 2017, 7.

35. Zhang Y, Zhang K, Fang A, Han Y, Yang J, Xue M, Bao J, Hu D, Zhou B, Sun X et al: Specific adaptation of Ustilaginoidea virens in occupying host florets revealed by comparative and functional genomics. Nat Commun 2014, 5:3849.

36. Song JH, Wei W, Lv B, Lin Y, Yin WX, Peng YL, Schnabel G, Huang JB, Jiang DH, Luo CX: Rice false smut fungus hijacks the rice nutrients supply by blocking and mimicking the fertilization of rice ovary. Environmental microbiology 2016, 18(11):3840-3849.

37. Cuesta Arenas Y, Kalkman ERIC, Schouten A, Dieho M, Vredenbregt P, Uwumukiza B, Osés Ruiz M, van Kan JAL: Functional analysis and mode of action of phytotoxic Nep1-like proteins of Botrytis cinerea. Physiological and Molecular Plant Pathology 2010, 74(5-6):376-386.

38. Kanneganti TD, Huitema E, Cakir C, Kamoun S: Synergistic interactions of the plant cell death pathways induced by Phytophthora infestans Nep1-like protein PiNPP1.1 and INF1 elicitin. Mol Plant Microbe Interact 2006, 19(8):854-863. 
39. Letunic I, Bork P: 20 years of the SMART protein domain annotation resource. Nucleic Acids Research 2017, 46(D1):D493-D496.

40. Gietz RD, Schiestl RH: Large-scale high-efficiency yeast transformation using the LiAc/SS carrier DNA/PEG method. Nature Protocols 2007, 2(1):38-41.

41. Yin W, Wang Y, Chen T, Lin Y, Luo C: Functional evaluation of the signal peptides of secreted proteins. Bio-protocol 2018, 8(9):e2839.

Figures 
MHRALLIAIAGINALSTKSQGASLETLDNVTFTIKGHPDLIVPPESLDAVSQGLCDDK GFDANPPPPLPQRATANDLKWQPALDFDTDSCYNVPAIGPDGHIDQGRSRHETNT EGCRDEYDLDHGNVYSRQRCNNGWCAYLYDYFFEKDIGDRICIGHQYDWEHLQV WTRNGEPQFGCASAHGKYKARLWKDLPKEGTHMKAVYNKDGLIGTHYFRFSKGA GDEPPENHKHVWWRSSLVSWNGFPTLHLRSKLMAYDFGAADIALSDAAFPKNLE QCAGQIKHKFADFKFDYTRDEGSPGSP

$\mathrm{B}$

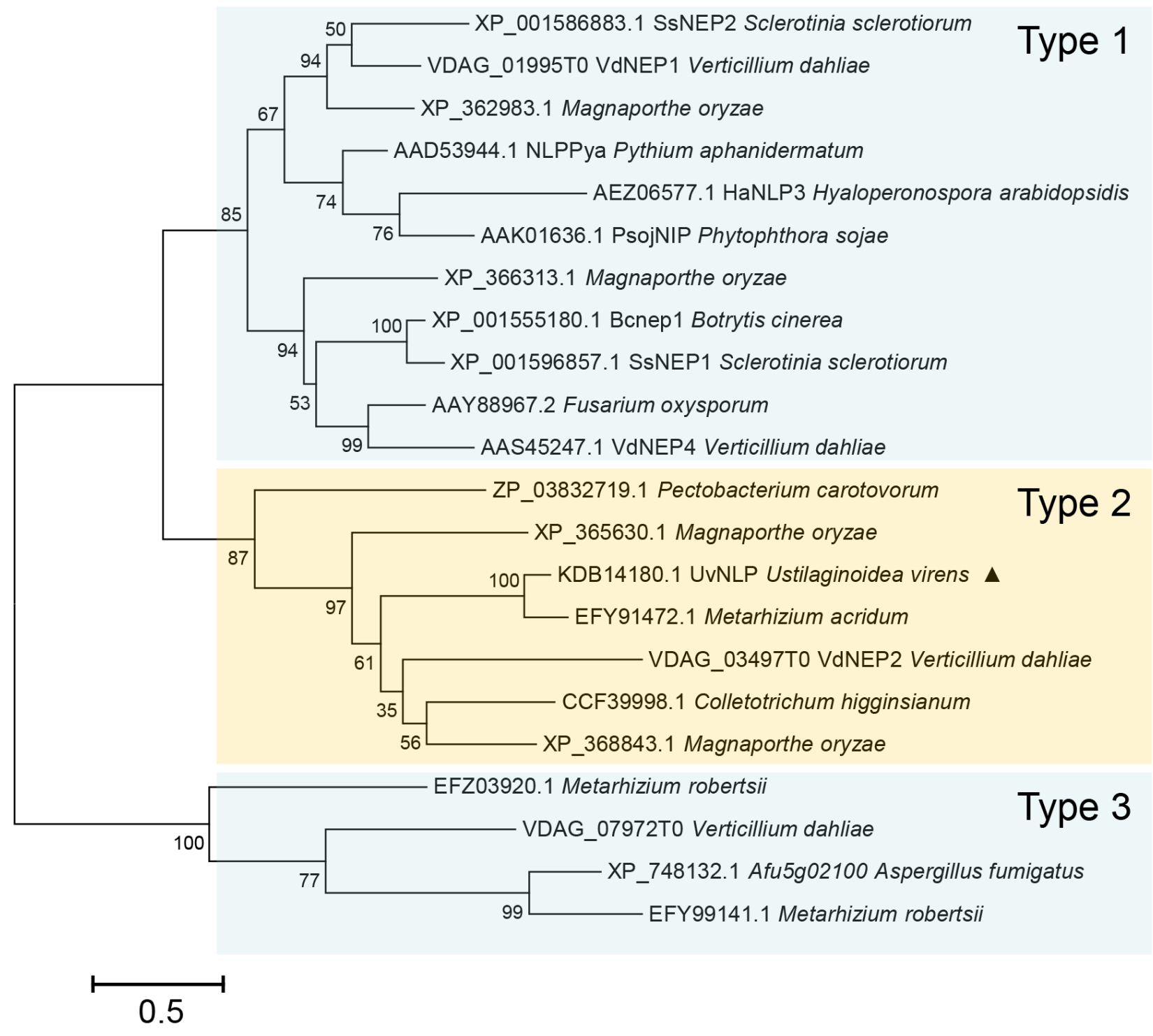

\section{Figure 1}

The amino acid sequence and phylogenetic analysis of UvNLP. (A) The amino acid sequence of UvNLP. The SP and NPP1 domains are underlined, and the conserved heptapeptide motif is indicated in red. (B) Phylogenetic analysis of UvNLP. The amino acid sequences were aligned with MEGA5, and a maximum likelihood tree was generated. UvNLP is indicated by a triangle. 


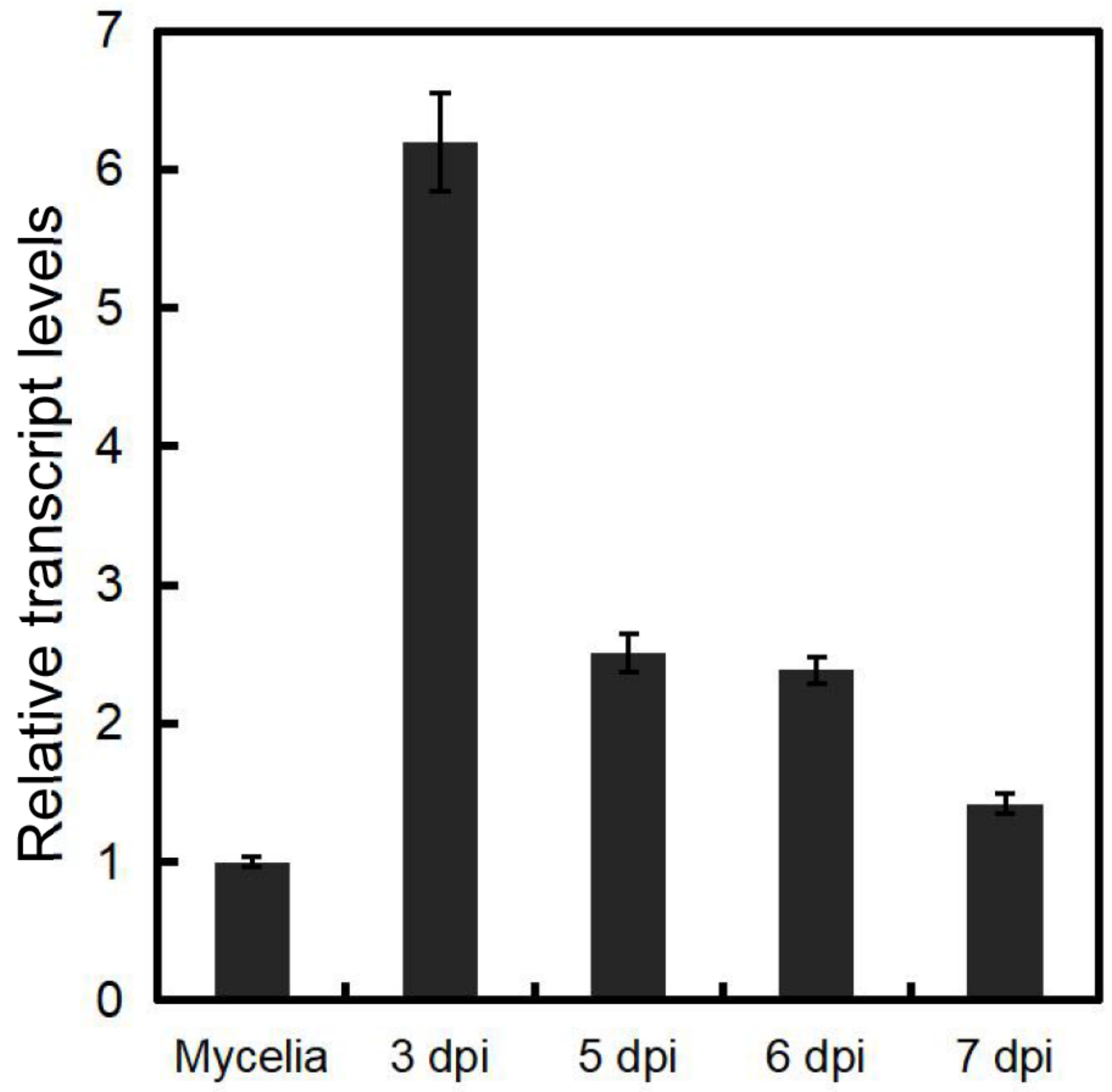

Figure 2

The transcript level of UvNLP at different stage. The expression of UvNLP was quantified using real-time PCR at 3, 5, 6 and 7 days post inoculation (dpi). Relative transcript level in the mycelia stage was regarded as the reference and normalized to 1 . 

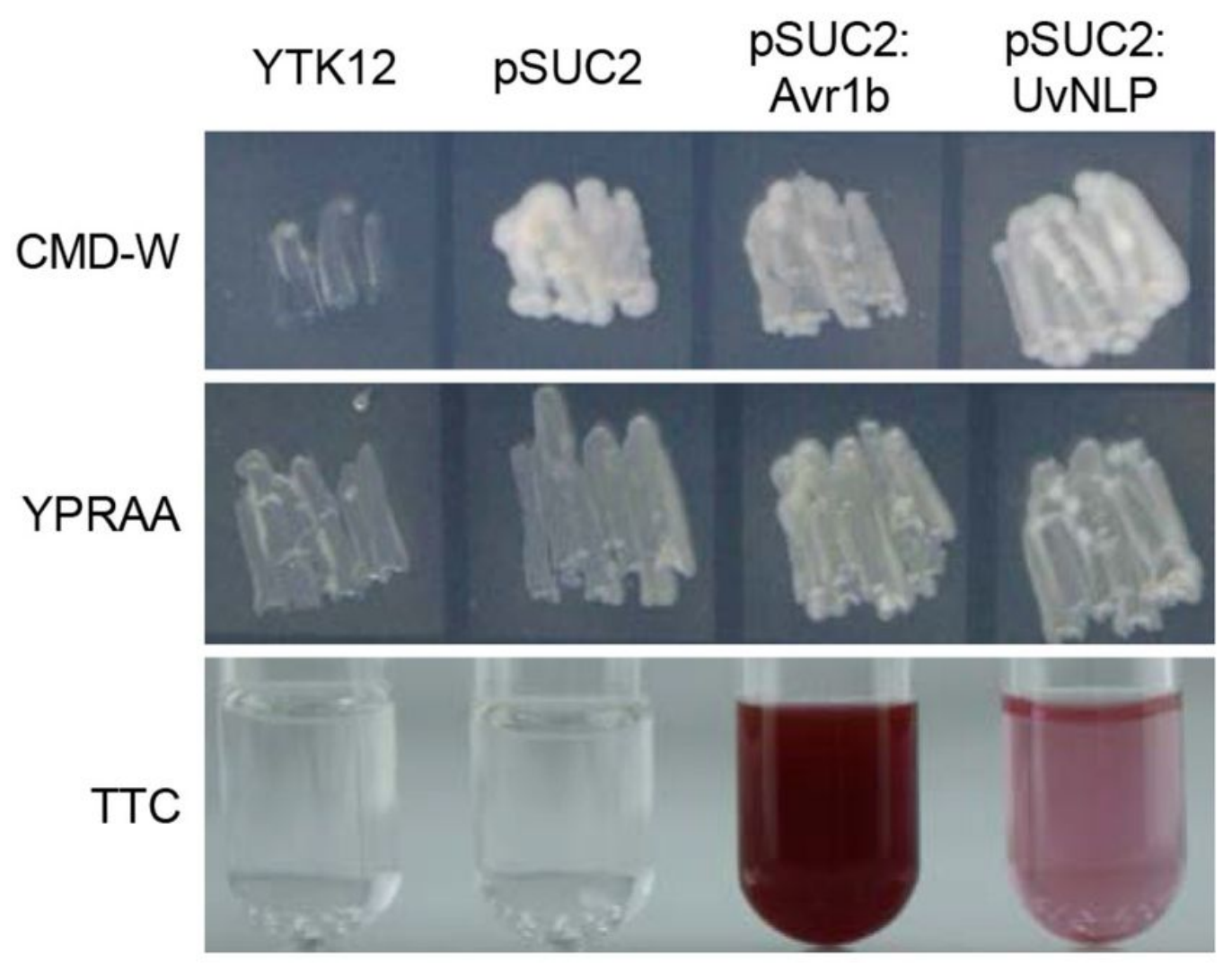

Figure 3

Yeast invertase secretion assay of the UvNLP signal peptide. The nucleotide sequence coding predicted signal peptide sequences and the following three amino acids (1-24) were constructed into the pSUC2 vector and transformed into the YTK12 strain. pSUC2 and pSUC2:Avr1b were used as the negative and positive controls. CMD-W was used to detect the yeast containing pSUC2, and YPRAA and TCC were used to confirm invertase secretion. 


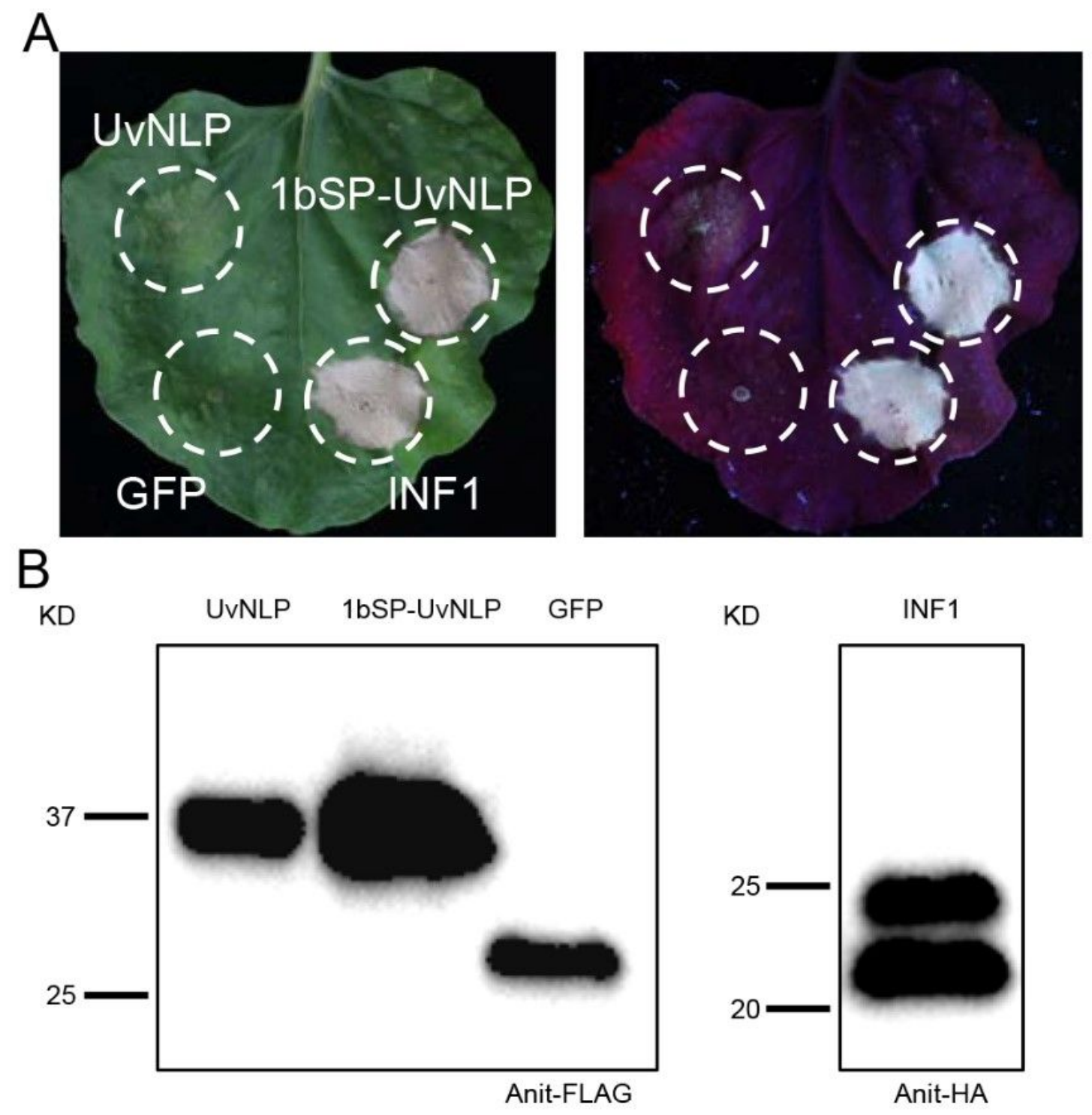

Figure 4

UvNLP induces weak cell death in N. benthamiana. (A) Phenotype observation after 7 days. The proteins were expressed using agrobacterium-mediated infiltration, and the phenotype was observed under light or UV light. (B) Western blot detection of expressed proteins. The proteins were extracted after 2 days of infiltration and detected using anti-FLAG or anti-HA antibody. 

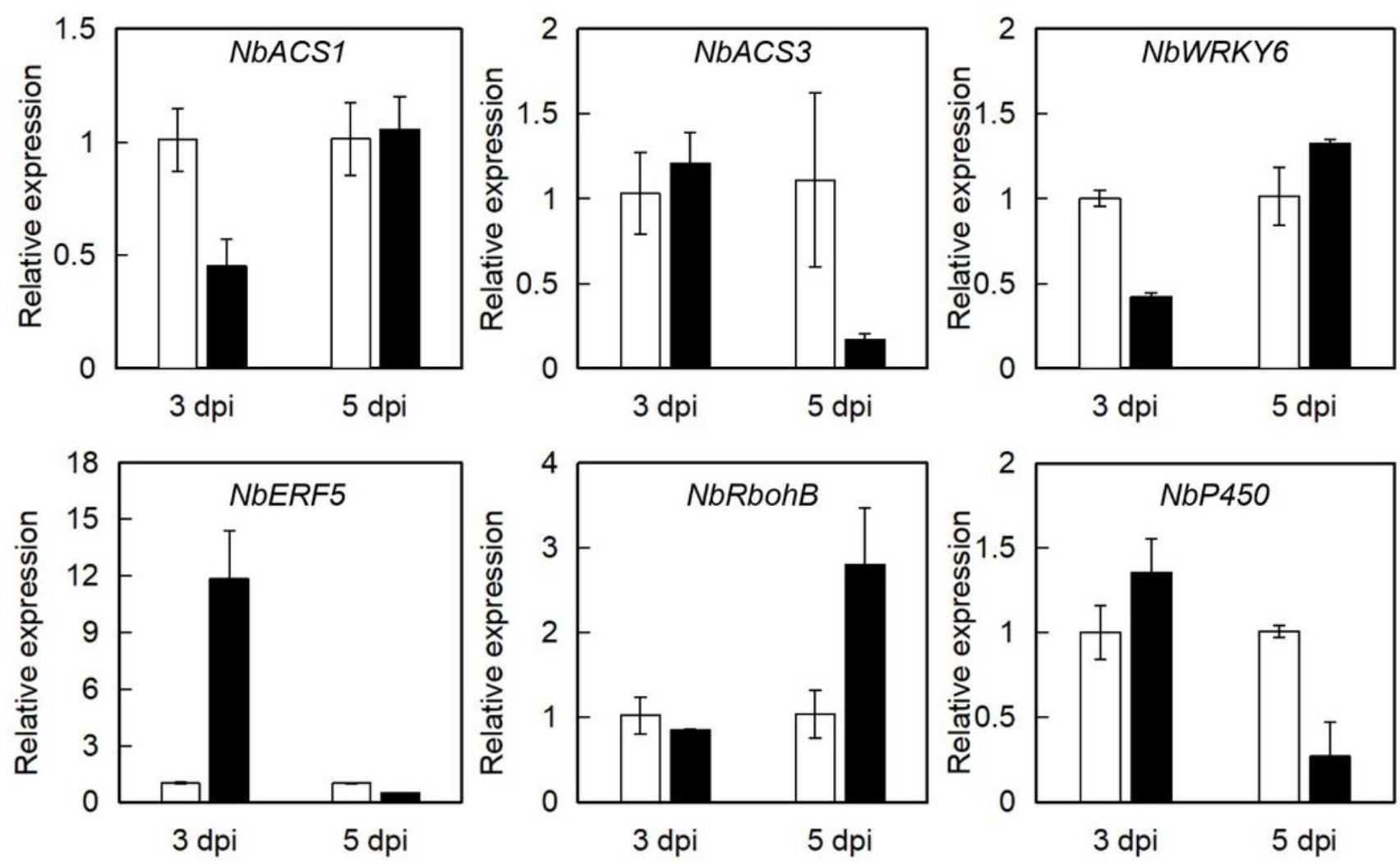

Figure 5

Transcription of resistance-related genes in $\mathrm{N}$. benthamiana. The relative transcript abundances of NbACS1, NbACS2, NbWRKY6, NbEFR5, NbRbohB and NbP450 in N. benthamiana plants 3 and 5 days post-infiltration. UvNLP (black) was normalized to GFP (white) which was assigned a value of 1.

\section{Supplementary Files}

This is a list of supplementary files associated with this preprint. Click to download.

- Additionalfile1Theprimersusedinthisstudy.doc 